\title{
Spindle assembly checkpoint-related failure perturbs early embryonic divisions and reduces reproductive performance of LT/Sv mice
}

\author{
Zuzanna Maciejewska ${ }^{1}$, Zbigniew Polanski ${ }^{3,4}$, Katarzyna Kisiel ${ }^{5}$, Jacek Z Kubiak ${ }^{6}$ \\ and Maria A Ciemerych ${ }^{2}$ \\ Departments of ${ }^{1}$ Embryology and ${ }^{2}$ Cytology, Institute of Zoology, Faculty of Biology, University of Warsaw, Ilji \\ Miecznikowa 1, 02-096 Warsaw, Poland, ${ }^{3}$ Department of Genetics and Evolution, Institute of Zoology, Jagiellonian \\ University, Ingardena 6, 30-060 Cracow, Poland, ${ }^{4}$ Department of Developmental Biology, Max Planck Institute of \\ Immunobiology, Freiburg D-79108, Germany, ${ }^{5}$ Animal Facility, Faculty of Biology, University of Warsaw, Warsaw, \\ Poland and ' 'Mitosis and Meiosis' Group, Institute of Genetics and Development, University of Rennes 1, CNRS-UMR \\ 6061, IFR 140 GFAS, 35043 Rennes, France
}

Correspondence should be addressed to M A Ciemerych; Email: ciemerych@biol.uw.edu.pl

J Z Kubiak; Email: jacek.kubiak@univ-rennes1.fr

Z Maciejewska is now at Molecular Basis of Carcinogenesis, Institut de Recherche sur le Cancer de Montpellier, Montpellier, France

\begin{abstract}
The phenotype of the LT/Sv strain of mice is manifested by abnormalities in oocyte meiotic cell-cycle, spontaneous parthenogenetic activation, teratomas formation, and frequent occurrence of embryonic triploidy. These abnormalities lead to the low rate of reproductive success. Recently, metaphase I arrest of LT/Sv oocytes has been attributed to the inability to timely inactivate the spindle assembly checkpoint (SAC). As differences in meiotic and mitotic SAC functioning were described, it remains obscure whether this abnormality is limited to the meiosis or also impinges on the mitotic divisions of LT/Sv embryos. Here, we show that a failure to inactivate SAC affects mitoses during preimplantation development of LT/Sv embryos. This is manifested by the prolonged localization of MAD2L1 on kinetochores of mitotic chromosomes and abnormally lengthened early embryonic M-phases. Moreover, LT/Sv embryos exhibit elevated frequency of abnormal chromosome separation during the first mitotic division. These abnormalities participate in severe impairment of preimplantation development and significantly decrease the reproductive success of this strain of mice. Thus, the common meiosis and mitosis SAC-related failure participates in a complex LT/Sv phenotype.

Reproduction (2009) 137 931-942
\end{abstract}

\section{Introduction}

The correct chromosome segregation during meiotic divisions of oocytes and spermatocytes, as well as during cleavage divisions of embryos, is one of the key factors determining successful development. Inaccuracies in these processes may result in anueploid gametes or embryonic cells. This usually leads to pregnancy loss or, occasionally, birth of individuals suffering from disorders caused by chromosomal aberrations (e.g. Down syndrome).

The spindle assembly checkpoint (SAC) helps to prevent aneuploidy by blocking anaphase onset until correct kinetochore-microtubule (MT) attachments and tensions are attained, and thus facilitates correct chromosome segregation. SAC comprises a multicomponent pathway, in which the transitory metaphase arrest depends upon the lack of correct kinetochore-MT attachment at least at one kinetochore (Musacchio \& Salmon 2007). Molecular mechanism of SAC activation engages localization of $B U B$ and MAD proteins to unattached kinetochores of both mitotic and meiotic chromosomes (Li \& Benezra 1996, Taylor \& McKeon 1997), and sequestering CDC20 - an activator of the anaphase promoting complex/cyclosome (APC/C; Fang et al. 1998). Recently, it was shown that ubiquitination and degradation of CDC20 via APC/C activity is necessary to maintain SAC activity (Nilsson et al. 2008). As APC/C itself accumulates preferentially at kinetochores of unattached chromosomes when SAC is activated (Acquaviva et al. 2004) it seems that the kinetochore localization of all these components can be critical for 
SAC activity. The establishment of stable MT-kinetochore connections leads to the inactivation of SAC and removal of BUB and MAD proteins from kinetochores. This results in CDC20 release and $A P C / C$ activation. Active $\mathrm{APC} / \mathrm{C}$ triggers ubiquitination and, in consequence, proteasome-dependent degradation of critical M-phase regulators. Among them are cyclin B - regulatory subunit of M-phase promoting factor (MPF; Draetta et al. 1989, Holloway et al. 1993) and PTTG1 (securin) responsible for cohesins stability necessary for chromatids cohesion (Marangos \& Carroll 2008). Ubiquitination of cyclin B targets the MPF complex to the proteasome which dissociates cyclin B from CDC2A. This is a critical point in CDC2A inactivation (Chesnel et al. 2006, 2007). Following this step, free cyclin B is degraded within the proteasome (Chesnel et al. 2006, 2007). Degradation of PTTG1 results in a cascade of events enabling chromatid separation (Uhlmann 2003). As a result, initiation of anaphase movement of chromosomes is coordinated with MPF inactivation and the cell division can be completed.

Oocytes from the LT/Sv inbred strain represent an interesting example of heritable abnormal meiosis, reduced reproductive fitness and pathology of development expressed by teratoma formation. In more detail, this complex phenotype includes the elevated susceptibility to undergo parthenogenetic activation (Kaufman \& Howlett 1986, Maleszewski \& Yanagimachi 1995, Ciemerych \& Kubiak 1998) that may result in ovarian teratomas (Stevens \& Varnum 1974, Eppig 1978b, Artzt et al. 1987, Eppig et al. 1996, Lee et al. 1997). Also, defective calcium release, which may be linked to spontaneous parthenogenesis, was demonstrated recently at fertilization of LT/Sv oocytes (Archacka et al. 2008). Another prominent feature of LT/Sv oocytes is their inability to complete meiotic maturation. A high proportion of in vivo and in vitro maturing LT/Sv oocytes does not progress to metaphase II (MII) but arrests in the first meiotic metaphase I (MI; Kaufman \& Howlett 1986, Hampl \& Eppig 1995, Maleszewski \& Yanagimachi 1995, Ciemerych \& Kubiak 1998). Such Ml-arrested oocytes are ovulated and upon fertilization give rise to non-viable triploid embryos (Kaufman \& Speirs 1987, O'Neill \& Kaufman 1987, Henery \& Kaufman 1993a). The molecular basis of the $\mathrm{MI}$ arrest of LT/Sv oocytes is not fully known. However, a recent study revealed that the SAC proteins BUB1 and MAD2L1 localize for prolonged time to the kinetochores during first meiotic M-phase indicating involvement of SAC activity in MI arrest (Hupalowska et al. 2008). Consistent with this observation, inactivation of the SAC pathway through expression of dominant negative mutant BUB1 resulted in release of the majority of $\mathrm{LT} / \mathrm{S} v$ oocytes from $\mathrm{MI}$ arrest (Hupalowska et al. 2008). Thus, the MI arrest of LT/Sv oocytes appears to be related to the inability to timely inactivate SAC (Hupalowska et al. 2008). The precise cause of SAC-related failure in LT/Sv strain of mice remains unknown.
The cell-cycle regulation of mouse oocytes and onecell embryos shares several similarities (Kubiak et al. 2008c). Both the meiotic maturation of the mouse oocyte and the first embryonic cell cycle occur in the absence of transcription, i.e., under the control of maternal factors accumulated during oocyte growth (Oh et al. 2000, Ma et al. 2001, Wang et al. 2004), with exception to certain rare transcripts appearing in the late zygote (Ram \& Schultz 1993, Bouniol et al. 1995). Both in the maturing oocyte as well as in the one-cell embryo the cell-cycle progression is controlled in a similar way by yet unidentified factors located in the cell nucleus (Polanski et al. 2005, Greda et al. 2006, Hoffmann et al. 2006, Mohammed et al. 2008). Last but not the least, certain discrete similarities in regulation of the M-phases in oocytes (MPF activation/inactivation and related phenomena as $M$-phase duration or cytoskeleton activities) and one-cell and two-cell embryos were also reported (Ciemerych et al. 1999, Kubiak \& Ciemerych 2001, Sikora-Polaczek et al. 2006). Therefore, the first mitotic cell cycle (and perhaps subsequent ones) is governed by specific mechanisms transitional between meiotic and mitotic type of cellcycle regulation (Kubiak et al. 2008a, 2008b, 2008c).

Thus, taking into consideration that meiotic factors may impinge on the embryonic mitoses it was possible that the hereditary defect affecting meiotic progression of LT/Sv oocytes influences also progression through the cell cycle of early LT/Sv embryos. We have previously shown that the meiotic defect of LT/Sv oocytes is related to SAC function (Hupalowska et al. 2008). Usually, SAC has a common mode of action in numerous eukaryotic cells studied so far. However, certain cell types may have modified checkpoint control depending on not yet well characterized factors. Mitotic versus meiotic differences are among the best known and were described for yeast, plant, and mammalian cells (LeMaire-Adkins et al. 1997, Yu et al. 1999, Kallio et al. 2000, LeMaire-Adkins \& Hunt 2000, Jeganathan \& van Deursen 2006, Lacefield \& Murray 2007, Yamamoto et al. 2008). Therefore, the issue of whether the LT/Sv defect impinging on SAC function in oocytes affects also cell-cycles of the early embryos remained fully open.

We show here that the SAC-related LT/Sv phenotype is not exclusively meiotic, but also impairs the progression of the early mitotic divisions of LT/Sv embryos. We demonstrate prolonged localization of MAD2L1 on the kinetochores of the one-cell and two-cell LT/Sv embryos and dramatic prolongation of these embryonic mitoses. Moreover, this correlates with anomalies often associated with SAC malfunction, like lagging chromosomes or presence of micronuclei. These abnormalities seem closely related to compromised preimplantation development and participate in the reduction of reproductive success of this strain of mice. 


\section{Results}

\section{Variable duration of the first embryonic mitosis in inbred strains of mice}

To determine whether SAC-related LT/Sv phenotype characterizing maturing oocytes (Hupalowska et al. 2008) also affects embryonic mitoses, we focused on the duration of the well characterized first mitosis (Sikora-Polaczek et al. 2006) in different strains of mice. First, we evaluated the range of the variability in duration of the first mitotic division in five different mouse strains: BALB/c, C3H, C57, DBA, and RI43. The latter one, i.e. RI43, is a recombinant inbred strain developed from the inbred strains CBA and KE (Golas et al. 2008). As in our previous studies concerning wildtype, either $\mathrm{F} 1(\mathrm{CBA} / \mathrm{H} \times \mathrm{C} 57 \mathrm{BI} / 10)$ or outbred Swiss albino mice, the length of the mitosis was delineated by the nuclear-envelope breakdown (NEBD) and the onset of anaphase, correlated with the subtle change in the cell shape as it entered cleavage (Ciemerych et al. 1998, 1999, Sikora-Polaczek et al. 2006). The duration of the first-embryonic mitosis was on average ( \pm s.D.) $116 \pm 14$, $112 \pm 21,100 \pm 22,90 \pm 19$ and $89 \pm 8 \mathrm{~min}$ in C57 $(n=7), \mathrm{DBA}(n=19), \mathrm{C} 3 \mathrm{H}(n=15), \mathrm{BALB} / \mathrm{c}(n=15)$ and RI43 ( $n=18)$ mice respectively. Statistical analysis (ANOVA) allowed us to distinguish the C57 and DBA strains as characterized by the 'long' first mitosis, differing significantly from BALB/C and RI43 strains, presenting the 'short' first-embryonic M-phase. One-cell embryos from $\mathrm{C} 3 \mathrm{H}$ mice exhibited intermediate M-phase duration, and did not differ statistically from embryos of other four strains (Fig. 1). For comparison, the length of the first-embryonic mitosis of $\mathrm{F} 1$ (CBA/H $\times \mathrm{C} 57 \mathrm{Bl} / 6$ ) hybrid mice, used in our previous studies on LT/Sv oocytes as wild-type control (Hupalowska et al. 2008), was $105 \pm 12 \min (n=14)$, which is in the middle of the range delineated by the five inbred strains tested.

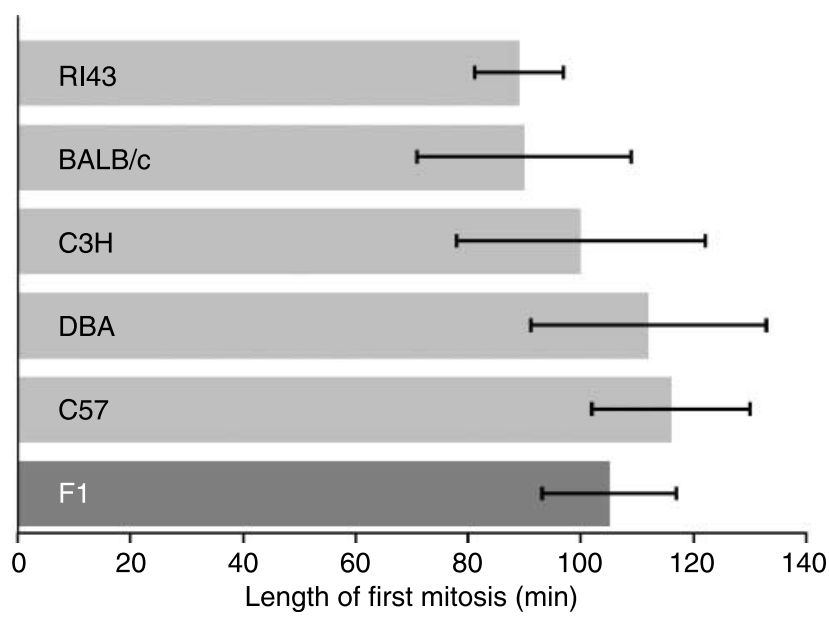

Figure 1 First-embryonic mitosis in different strains of mice. The length of mitoses was analyzed in embryos of RI43, BALB/C, C3H, DBA, and $\mathrm{C} 57$ strains of mice, as well as in $\mathrm{F} 1(\mathrm{CBA} / \mathrm{H} \times \mathrm{C} 57 \mathrm{Bl} / 6)$ embryos.

\section{First mitosis of LT/SV embryos is enormously prolonged}

We analyzed the duration of the first mitosis in LT/SV in parallel with the control, wild-type $\mathrm{F} 1(\mathrm{CBA} / \mathrm{H} \times \mathrm{C} 57 \mathrm{Bl} / 6)$ one-cell parthenogenetic embryos. We decided to use parthenogenetic one-cell embryos because, as shown previously, they undergo NEBD more synchronously than fertilized ones (Sikora-Polaczek et al. 2006). Moreover, we previously showed that the timing of MPF activation, spindle formation, mitosis duration, and the most importantly SAC inactivation do not differ between onecell parthenogenotes and fertilized embryos (Ciemerych et al. 1999, Sikora-Polaczek et al. 2006). Other studies also confirmed the usefulness of one-cell parthenogenotes for the analyses of different aspects of the mouse preimplantation development (Waksmundzka et al. 1984, Borsuk \& Tarkowski 1989, Ciemerych 1995, Winston \& Maro 1995, Kalab et al. 1996). However, time-lapse recordings were performed using exclusively embryos obtained after in vivo fertilization, i.e., zygotes and two-cell embryos. Since parthenogenetic and fertilized one-cell embryos did not differ in the timing of first mitosis, we present the pooled data obtained from both types of embryos.

The first mitosis of wild-type embryos took on average $105 \pm 12$ min ( $n=14$, Figs 1 and 2 A) consistent with the previous data (Ciemerych et al. 1999, Sikora-Polaczek et al. 2006). In LT/Sv embryos, however, the first mitosis took on average ( \pm s.D.) $183 \pm 57 \mathrm{~min}(n=53$; Fig. $3 \mathrm{~A})$. The length of the shortest of these mitoses was similar to those of onecell F1 embryos, and was $115 \mathrm{~min}$. However, the longest one took as long as $345 \mathrm{~min}$ (Fig. 3B). Thus, the length of the first mitosis in LT/Sv embryos seems to be extremely long beyond the length of the typical mitosis in the wild-type mice. Moreover, although we found that the length of the first mitosis can differ reasonably depending on the genetic background (Fig. 1), the duration of the process in the LT/Sv embryos exceeded greatly the range delineated by the six types of randomly chosen genotypes differing significantly both from F1 mice (Fig. $3 \mathrm{~A}$ ) and from all five inbred strains tested $(P<0.0001$ in all six cases, ANOVA).

It is important to remind that although the majority of LT/Sv oocytes are ovulated in the MI stage, some proceed to the MII before ovulation (Kaufman \& Howlett 1986, O'Neill \& Kaufman 1987). Thus, the great variability in the length of the first mitosis of LT/Sv embryos (Fig. 3B) may in fact reflect the heterogeneity of embryos tested, i.e. originating from $\mathrm{Ml}$ and MII oocytes. To address this issue, in a separate experiment, we first classified ovulated oocytes into MI or MII group and measured duration of the first mitosis in parthenogenetic embryos originating from each group of oocytes. Interestingly, the duration of the first mitosis did not differ significantly regardless of the 'origin' of one-cell parthenogenetic 


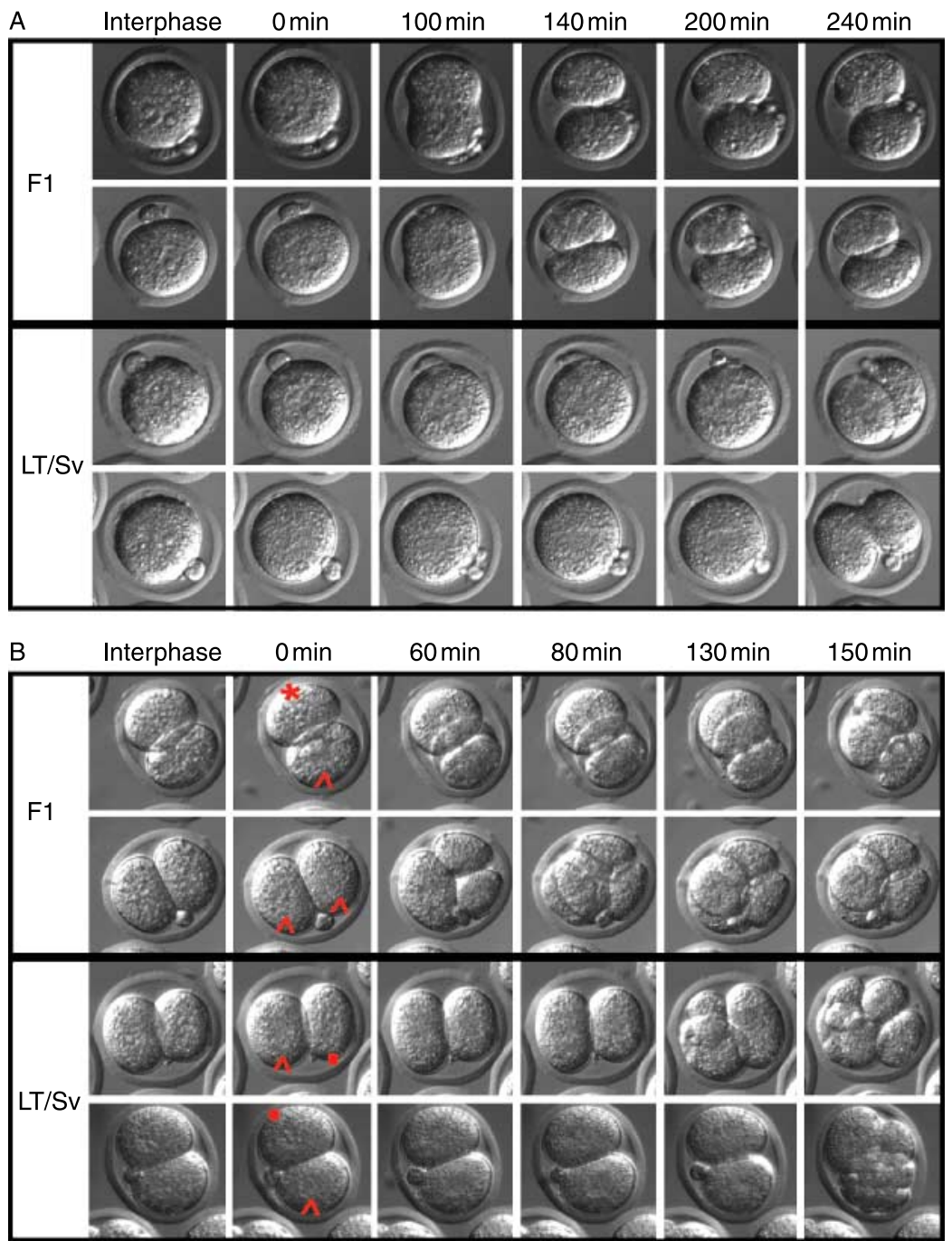

Figure 2 Time-lapse recording of the first and the second division of $\mathrm{F} 1(\mathrm{CBA} / \mathrm{H} \times \mathrm{C} 57 \mathrm{BI} / 6)$ and $\mathrm{LT} / \mathrm{Sv}$ embryos. (A) Examples of one-cell embryos (zygotes) progressing through the first mitosis. (B) Examples of two-cell embryos progressing through mitosis.

Blastomeres undergoing NEBD exactly at time 0 min are marked with red arrowheads. The F1 blastomere marked with a red asterisk underwent NEBD at time $80 \mathrm{~min}$. LT/Sv blastomeres marked with red boxes underwent NEBD at time $5 \mathrm{~min}$. Due to space limitations, instead of the whole recording only the selected images are shown. embryos (Fig. 3C). Thus, 'escaping' MI during oocyte meiosis does not guarantee the normal progression of LT/ Sv embryos throughout the first mitotic division.

\section{Second mitosis of LT/SV embryos is also prolonged in comparison with the wild-type mice}

The second mitosis was investigated in blastomeres of two-cell embryos obtained by in vivo fertilization, similarly as described previously (Ciemerych et al. 1999, Sikora-Polaczek et al. 2006). In control, F1 embryos this cleavage division took on average 77 \pm 10 min $(n=19)$ consistently with the data presented previously (Ciemerych et al. 1999, Sikora-Polaczek et al. 2006)), while in LT/Sv embryos it took $140 \pm 40 \mathrm{~min}$ $(n=37)$. Similarly, as it was observed for the first mitosis, the length of second mitoses varied enormously, i.e. from 78 to $235 \mathrm{~min}$ (Figs 2B and 3B). Thus, the average first or second mitotic division was significantly prolonged in LT/Sv embryos, as compared with F1 embryos. Notably, although the prolonged M-phase significantly delayed the exit from the first and the second mitosis, embryos have never arrested permanently during these divisions. These data indicate that the prolonged $\mathrm{M}$-phases are specific feature of at least two first-embryonic mitoses of the LT/Sv mice.

\section{SAC activity is dramatically prolonged during the first mitotic division of LT/SV embryos}

The prolongation of SAC activity during meiotic maturation of LT/Sv oocytes led to the MI arrest (Hupalowska et al. 2008). Thus, we investigated whether the sustained SAC activity plays a role also in the abnormal regulation of the first two embryonic mitoses of this strain of mice. To this point, we compared the presence or absence of MAD2L1 at kinetochores in F1 and LT/Sv embryos. Previously, we documented that in F1 embryos MAD2L1 was lost from kinetochores $\sim 20 \mathrm{~min}$ after NEBD during the first 
mitosis, and $\sim 30$ min after NEBD during the second one (Sikora-Polaczek et al. 2006). Accordingly, $60 \mathrm{~min}$ after NEBD none of the F1 one-cell embryos $(n=14)$ contained MAD2L1 at kinetochores (Fig. 4A). However, in all one-cell LT/Sv embryos analyzed at $30 \mathrm{~min}(n=13)$ and 60 min after NEBD $(n=15)$ MAD2L1 was detectable at kinetochores (Fig. 4B). At $90 \mathrm{~min}$ after NEBD two out of eight embryos still had MAD2L1 localized at kinetochores. At $120 \mathrm{~min}$ after NEBD in all analyzed LT/Sv embryos $(n=8)$ MAD2L1 was absent from kinetochores, indicating that they entered metaphase after prolonged prometaphase. Thus, the delay of SAC inactivation is not limited exclusively to meiosis, as revealed recently (Hupalowska et al. 2008), but also to the early mitotic divisions of LT/Sv embryos.

\section{Early LT/Sv embryos exhibit chromosome segregation errors and impaired development}

Next, we investigated whether the problems with timely SAC inactivation and delayed mitotic anaphase onset in embryos correlate with the anomalies in chromosome
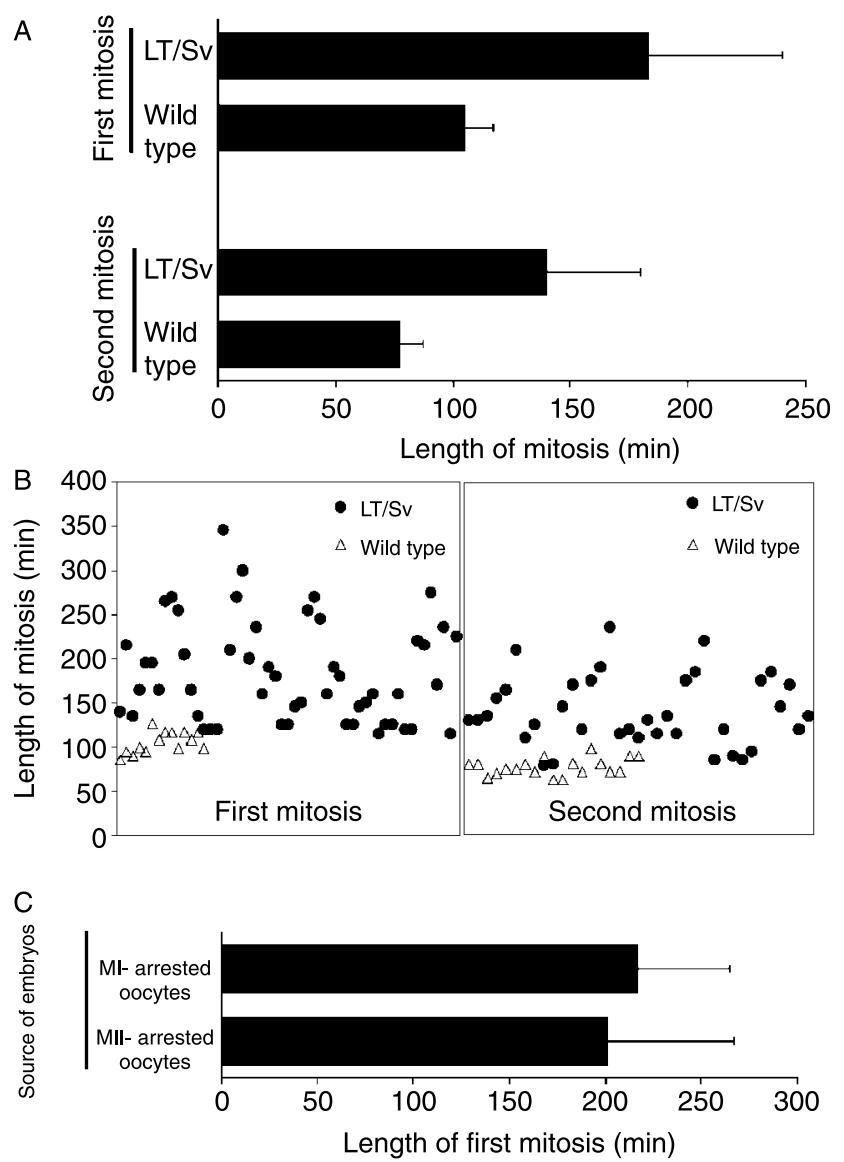

Figure 3 First and second mitosis is prolonged in LT/Sv embryos. (A) Average length of mitosis in wild-type and LT/Sv embryos. For both mitoses, the differences between LT/Sv and F1 embryos are statistically significant (t-test). (B) Scatters showing variability in the duration of mitoses. (C) Average length of first mitosis in LT/Sv embryos originated from MI and MII oocytes.
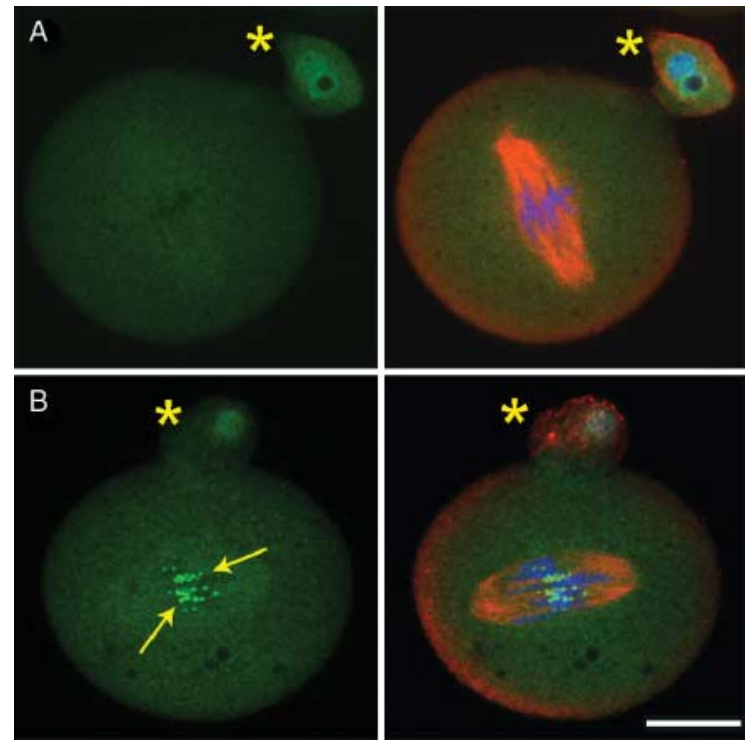

Figure 4 Localization of MAD2L1 in wild-type and LT/Sv one-cell embryos during the first mitotic division. (A) Wild-type one-cell embryo fixed 60 min after NEBD, no MAD2L1 visible at kinetochores. (B) LT/Sv oocyte fixed 60 min after NEBD, MAD2L1 localized at kinetochores. Left panel: MAD2L1-green, right panel: overlay of MAD2L1, tubulin - red and chromatin labeled with chromomycine blue. Asterisks indicate polar body, arrows indicate MAD2L1 localization in kinetochore region. Bar $20 \mu \mathrm{m}$.

segregation as observed in oocytes (Hupalowska et al. 2008). All but one of the control F1 embryos fixed in telophase of the first mitotic division $(n=19)$ and all fixed in the two-cell stage $(n=40)$ contained either correctly separating chromosomes (Table 1 and Fig. 5A) or morphologically normal interphase nuclei (Table 1 and Fig. 5B). However, this was not the case in LT/Sv embryos. Similarly, as during induced meiotic anaphase I/telophase I in LT/Sv oocytes (Hupalowska et al. 2008), anomalous chromosome separation was also detectable in embryos of this strain (Table 1). One out of five dividing one-cell embryos had abnormally positioned chromosomes (Fig. 5C). Moreover, 3 out of 24 analyzed two-cell embryos contained micronuclei documenting aberrant chromosome separation (Fig. 5D). Statistical analysis using Fisher's exact probability test, which is an excellent choice for small samples, revealed that the frequency of abnormal chromosome arrangements related to errors during the first embryonic division is clearly higher and statistically important in LT/Sv embryos when compared with wild-type ones (Table 1).

Further comparisons of control F1 and LT/Sv embryos obtained after in vivo fertilization revealed that not only the first but also subsequent cleavages proceed incorrectly. Analysis of ten wild-type embryos fixed at $80 \mathrm{~h}$ after hCG, i.e. at the morula stage, showed that they contained $\sim 14$ blastomeres per embryo (Fig. 5E and F). By contrast, such analysis of 12 simultaneously cultured $\mathrm{LT} / \mathrm{S} v$ embryos revealed severely decreased cell number, 
Table 1 Frequency of chromosomal abnormalities related to the errors during first-embryonic division in the embryos from LT/Sv and wildtype mice.

\begin{tabular}{|c|c|c|c|}
\hline \multirow[b]{2}{*}{ Embryos } & \multicolumn{3}{|c|}{ Type of abnormalities } \\
\hline & $\begin{array}{c}\text { Aberrant } \\
\text { chromosome } \\
\text { separation }\end{array}$ & $\begin{array}{l}\text { Micronuclei at } \\
2 \text {-cell stage }\end{array}$ & $\begin{array}{c}\text { Total } \\
\text { abnormalities }\end{array}$ \\
\hline Wild-type & $1 / 19(5 \%)$ & $0 / 40(0 \%)^{*}$ & $1 / 59(2 \%)^{*}$ \\
\hline $\mathrm{LT} / \mathrm{Sv}$ & $1 / 5(20 \%)$ & $3 / 24(12.5 \%)^{*}$ & 4/29 (14\%)* \\
\hline
\end{tabular}

*Difference between LT/Sv and wild-type embryos statistically significant $(P<0.05$, Fisher exact probability test).

which varied from two blastomeres in the less advanced embryos, up to nine blastomeres in the most advanced ones (Fig. 5G and $\mathrm{H}$ ). Moreover, this feature was accompanied by the presence of multinucleated blastomeres and blastomeres containing micronuclei (Fig. 5G and $\mathrm{H}$ ). This observation clearly suggested deregulation of mitotic divisions of preimplantation LT/Sv embryos.

To study the potential impact of these abnormalities on the preimplantation embryo development 193 wild-type embryos and $63 \mathrm{LT} / \mathrm{Sv}$ embryos were cultured from onecell stage ( $24 \mathrm{~h}$ after hCG) until the blastocyst stage $(94 \mathrm{~h}$ after hCG). A group of 153 wild-type and of 25 LT/Sv embryos were cultured for additional $24 \mathrm{~h}$ i.e., until $\sim 120 \mathrm{~h}$ after hCG. During the whole culture period LT/Sv embryos showed significant delay in their development (Fig. 6). Thus, not only the first and the second cleavage divisions were delayed as we described above, but also the subsequent ones, resulting in the retarded development of LT/Sv embryos. In consequence, while by $120 \mathrm{~h}$ after hCG the majority of wildtype embryos reached expanded blastocyst stage, a significant proportion of LT/Sv embryos were found at the morula stage and also a significant proportion underwent degeneration (Fig. 6). Such a hampered preimplantation development seems a logical consequence of the elevated frequency of abnormal chromosome separation in LT/Sv embryos.

To correlate the developmental problems observed during preimplantation period with the level of reproductive success of LT/Sv strain, we analyzed the size of litters of individual mating females of this strain and F1 controls. This analysis revealed that while all of the wildtype females $(n=68)$ gave birth to live progeny at least two times, $31 \%(21 / 68)$ of LT/Sv females were infertile. Moreover, the average number of newborns in the first two litters was significantly reduced in LT/Sv females (Table 2).

\section{Discussion}

We document here that the prolonged SAC activity is manifested by extended MAD2L1 presence on kinetochores and that it prolongs early mitotic M-phases,
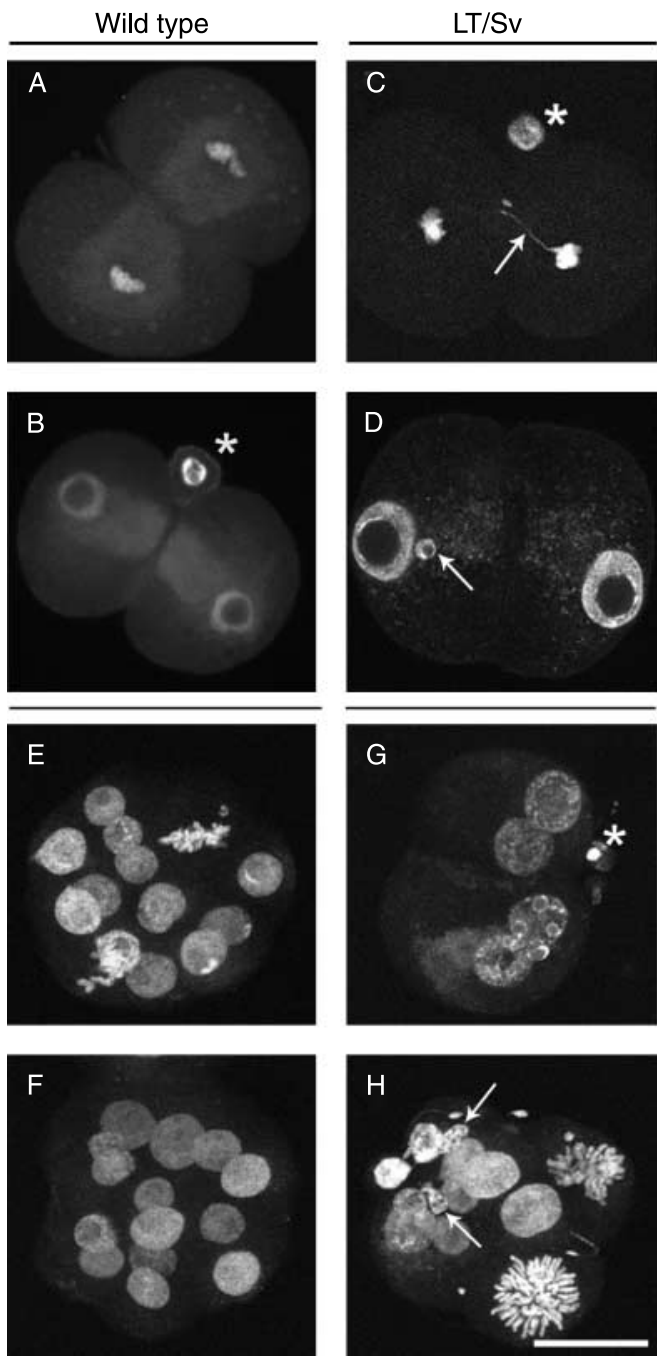

Figure 5 Chromatin organization in cleaving wild-type and LT/Sv embryos. (A) Wild-type embryo fixed at the telophase of the first mitotic division. (B) Wild-type embryo fixed at two-cell stage. (C) LT/Sv embryo fixed at the telophase of the first mitotic division, arrow indicates incorrectly separating chromosome. (D) LT/Sv embryo fixed at the twocell stage, arrow indicates the micronucleus resulting from incorrectly separated chromosome or chromosomes. (E and F) Wild-type embryos at the morula stage composed of 14 blastomeres, fixed $80 \mathrm{~h}$ after hCG. (E) Two blastomeres undergo mitotic division. ( $\mathrm{G}$ and $\mathrm{H}$ ) Abnormally dividing LT/Sv embryos fixed $80 \mathrm{~h}$ after hCG. (G) Embryo arrested in two-cell stage with multinucleated blastomeres. $(\mathrm{H})$ Embryo containing mitotically dividing blastomeres with abnormally high number of chromosomes and blastomeres containing micronuclei. Asterisks indicate polar body; arrows indicate abnormally positioned chromosomes or micronuclei. Bar $20 \mu \mathrm{m}$.

perturbs cleavage divisions, hampers preimplantation development, and correlates with the decreased reproductive success of LT/Sv mice. The SAC-related anomaly is certainly not the sole reason for the low reproductive performance of LT/Sv females. It coincides with the $\mathrm{MI}$-arrest of oocytes, the development of ovarian teratomas (Stevens \& Varnum 1974), spontaneous parthenogenetic activation of ovulated oocytes (Stevens 


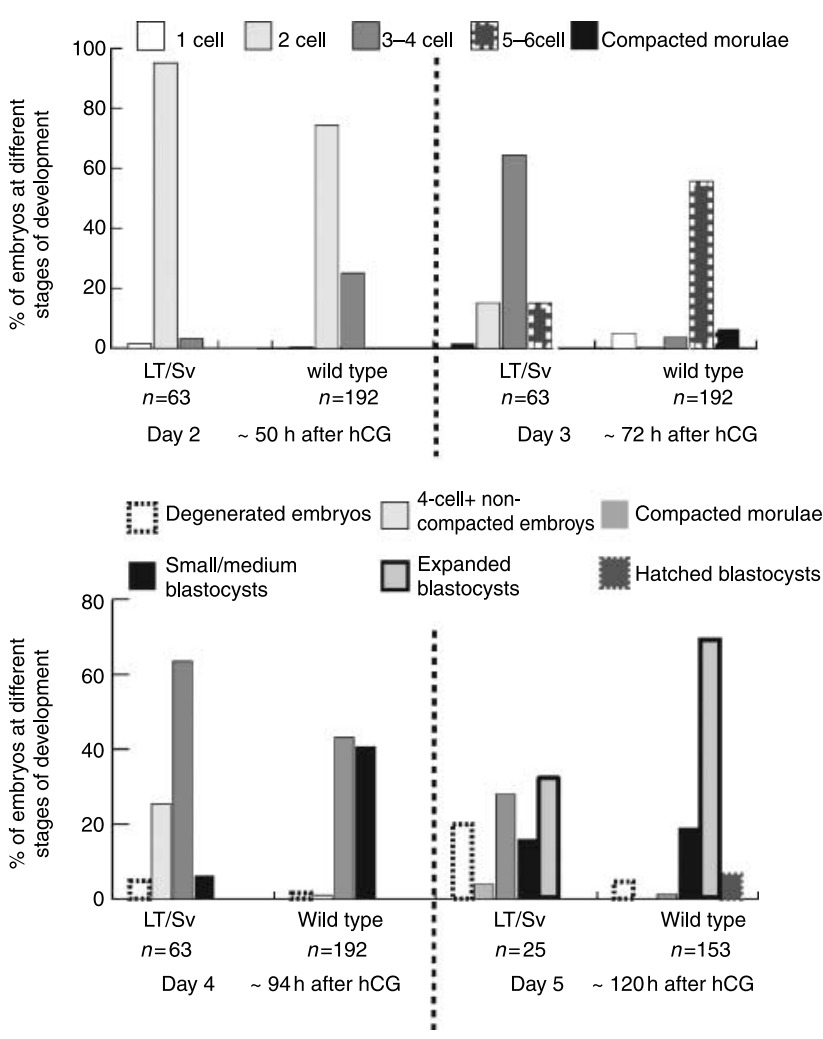

Figure 6 In vitro development of wild-type and LT/Sv embryos. Graphs show the proportion of wild-type and LT/Sv embryos in different stages of development during in vitro culture. Embryos were isolated at $24 \mathrm{~h}$ after hCG and scored at 50, 72, 94, and $120 \mathrm{~h}$ after hCG.

\& Varnum 1974, Eppig 1978a), or formation of triploid embryos upon fertilization (Kaufman \& Speirs 1987, Speirs \& Kaufman 1988, Henery \& Kaufman 1993a). As a result $30 \%$ of LT/Sv females do not reproduce at all and the remaining ones show a significant decrease in fertility (this study). It has to be noted, however, that triploidy does not affect the preimplantation development of wild-type mouse embryos that are able to reach the blastocyst stage and do not exhibit SAC-related phenotype of LT/Sv embryos described in our paper. (Niemierko 1975, Henery \& Kaufman 1992, 1993b).

We show here that the length of the first and second mitosis in LT/Sv embryos is enormously prolonged. The prolonged presence of MAD2L1 on the kinetochores of $\mathrm{LT} / \mathrm{S} v$ embryos strongly suggests that the extension of mitotic division result from the inability to timely inactivate the SAC. In our previous study, we have shown that in the wild-type one-cell embryo (the same F1 hybrids as used in the present study) MAD2L1, whereas present on the kinetochores until 10 min after NEBD, is lost from the kinetochores of all analyzed embryos before $20 \mathrm{~min}$ after NEBD (Sikora-Polaczek et al. 2006). Since, on average, the one-cell embryos of F1 mice (wild-type) divide $105 \mathrm{~min}$ after NEBD, we estimate that, on average, 85 min elapses from the release of the kinetochore-located MAD2L1 to the cleavage (105-20 min; Hupalowska et al. 2008). On the other hand, in the LT/Sv mice MAD2L1 is present on kinetochores of all tested one-cell embryos $60 \mathrm{~min}$ after NEBD and in 25\% of embryos even 90 min after NEBD. Thus, in one-cell LT/Sv embryos, the estimated time elapsing from the release of MAD2L1 from kinetochores to the cleavage is $\sim 93 \mathrm{~min}$ (183 $\mathrm{min}$ of average duration of the first mitosis $-90 \mathrm{~min}$ ) being very similar to this calculated for F1 control (93 vs $85 \mathrm{~min}$ ). Thus, the prolongation of the first mitosis in LT/Sv embryos seems indeed to occur thorough the extension of this phase of mitosis when MAD2L1 localizes to kinetochores. This strongly suggests that the enormously long mitosis of LT/ Sv embryos is exclusively SAC-dependent.

By evaluating the length of mitosis in the five randomly chosen inbred strains, we have found that the timing of the first mitosis can differ reasonably in embryos of different genotype. Previously, we estimated the timing of the first meiotic M-phase of oocytes (calculated as the time elapsing from the germinal-vesicle breakdown, which is an equivalent of NEBD in one-cell embryos, until cleavage) for four of these strains (Polanski 1997). As shown in Table 3, there is no correlation between the duration of the first meiotic M-phase and the duration of the first-embryonic mitosis in these four strains. However, in the LT/Sv mice the extremely prolonged first meiotic M-phase correlates with extremely extended first mitosis. This strongly suggests that in the LT/Sv oocytes and early embryos the defect is specific and common for meiosis and mitosis. Other characteristic $\mathrm{LT} / \mathrm{Sv}$ anomalies, like prolonged presence of MAD2L1 on the kinetochores or a tendency to abnormal chromosome separation, are observed both at meiosis (Hupalowska et al. 2008) and mitosis (present study). This delivers an additional support for the hypothesis that the defect in the oocytes and early embryos of LT/Sv mice may have the same origin.

Table 2 Comparison of fertility of LT/Sv and wild-type mice.

\begin{tabular}{lcccc}
\hline & & \multicolumn{2}{c}{ No. of young in } \\
Mice & No. of females & No. of fertile females $(\%)^{*}$ & 1 st litter & $2 \mathrm{nd} \mathrm{litter}$ \\
\hline Wild-type & 68 & $68(100)^{\mathrm{a}}$ & $8.1 \pm 3.7^{\mathrm{b}}$ & $7.0 \pm 2.5^{\mathrm{c}}$ \\
LT/Sv & 68 & $47(69)^{\mathrm{a}}$ & $4.7 \pm 1.8^{\mathrm{b}}$ & $4.2 \pm 2.0^{\mathrm{c}}$ \\
\hline
\end{tabular}

${ }^{a, b, c}$ Values marked with the same letter differ significantly (in all cases $P<0.0001,{ }^{a} \chi^{2}$ test of independence, ${ }^{b, c} t$-test).

* Delivering at least one litter. ${ }^{+}$Infertile females excluded. 
Table 3 The duration of the first meiotic M-phase and the first mitosis in different mouse inbred strains.

\begin{tabular}{lcl}
\hline Strains order & Duration $(\mathrm{min})$ & Phenotype \\
\hline Length of first meiosis & & \\
DBA & 384 & Fast \\
C3H & 516 & Intermediate \\
Balb/c & 522 & Intermediate \\
C57 & 528 & Intermediate \\
KE & 588 & Slow \\
Length of first mitosis & & \\
Balb/c & 90 & Fast \\
C3H & 100 & Intermediate \\
DBA & 112 & Slow \\
C57 & 116 & Slow \\
\hline
\end{tabular}

The duration of meiotic M-phase is calculated as the time elapsing between germinal vesicle breakdown (GVBD, an equivalent of NEBD in embryos) and the first polar body extrusion (equivalent of mitotic cleavage). Strains are ordered from the shortest meiosis/mitosis to the longest one. KE strain (which due to extinction is no longer available) is shown to indicate that there exists the slow meiotic phenotype. Data for meiosis are taken from Polanski (1997).

SAC activity results from incorrect attachment between kinetochores and MTs. However, no apparent morphological differences in mitotic spindle architecture were found between maturing LT/Sv and control embryos. The only spindle malformation in LT/Sv mice was observed in MI-arrested LT/Sv oocytes as a characteristic increase in size of the meiotic spindle (Albertini \& Eppig 1995, Ciemerych \& Kubiak 1998). It cannot be excluded that the failure to timely inactivate SAC during embryonic mitoses could arise from discrete (and morphologically undetectable at the light microscopy level) anomalies in MT attachment to kinetochores during the spindle formation. Interestingly, in Ptk1 cells the depletion of NDC80 (HEC1) protein (involved in MT attachment to kinetochores) results in concomitant events: i) disorganization of the mitotic spindle often linked to the increase in its size, ii) a $40 \mathrm{~min}$ delay in anaphase, and iii) a decrease in MAD2L1 recruitment to the kinetochores (Martin-Lluesma et al. 2002, Guimaraes et al. 2008). NDC80 depletion in Ptk1 cells shows that SAC activity can be only temporarily prolonged with discrete morphological changes in the spindle, which resembles the phenotype observed in LT/Sv oocytes and embryos. Alternatively, the SAC defect in LT/Sv embryos may rely on a hampered SAC pathway itself, for example an incorrect and/or delayed transmission of signals in SAC regulatory system.

It is commonly acknowledged that the basic SAC mechanism acts similarly during both mitotic and meiotic division. However, mitotic and meiotic cellular environments differ significantly and modify SAC action. Most importantly, in oocytes, in contrast to embryos, the MOS/.../EMI2 (FBXO43) pathway inhibits APC/C in a similar way as does the SAC (Kubiak et al. 2008C). This results in a doubled system of APC/C inhibition upon MII arrest. To our knowledge, consequences of such apparently redundant inhibitory control of anaphase trigger were not studied in details so far. For this major cause, SAC deregulation may differently influence female meiosis and mitosis (Pesin \& Orr-Weaver 2008).

It has been shown that MAD2L1, BUB3, BUB1B deficiency is fatal both for mouse embryogenesis and for the viability of somatic cells (Dobles et al. 2000, Kalitsis et al. 2000, Michel et al. 2001, Baker et al. 2004, Jeganathan \& van Deursen 2006). As far as meiosis is concerned, ablation of BUB1B causes the failure of meiotic division (Baker et al. 2004, 2005). Loss of one Mad2/1 allele results in significantly increased aneuploidy rate in MII arrested mouse oocytes, indicating that a single Mad2/1 gene copy lowers the fidelity of the meiotic division (Niault et al. 2007). Both knockdown of MAD2L1 as well as interfering with BUB1 function result in premature $\mathrm{MI} / \mathrm{MII}$ transition (Tsurumi et al. 2004, Homer et al. 2005). However, despite the involvement of the same key proteins in mitotic/meiotic SAC function some intriguing issues related to SAC remain. For example, deficiency of some SAC proteins was not shown to influence the course of meiosis and does not result in premature chromatid segregation (Jeganathan \& van Deursen 2006, Pesin \& Orr-Weaver 2008). Also the progression of meiosis in female $X O$ mice is not affected by the presence of univalent chromosome X (LeMaire-Adkins et al. 1997), suggesting that the specific $X$-univalent structure permits the establishment of bi-oriented connections to the meiotic spindle. Furthermore, while mitotically-dividing cells arrest in prometaphase in the response to the presence of MT-disassembling drugs, the meiotically dividing mouse spermatocytes arrest at pachytene (Tepperberg et al. 1999). This suggests the presence of specific SAC effectors also during male meiosis. In meiotically dividing budding yeast in which chromosome exchange is affected, MAD3/BUB1B appears crucial for delaying prometaphase I rather than metaphase-anaphase transition (Cheslock et al. 2005). This, again, suggests a unique role of checkpoint proteins in meiotic progression. Aware of these differences the characteristics of mitotic SAC-related defects described in our present study resemble very closely the meiotic phenotype of LT/ Sv oocytes (Hupalowska et al. 2008). Thus, it seems highly possible that the defects in SAC functioning observed in oocytes and early embryos might have the same molecular basis. We did not, however, address the question about the molecular mechanism underlying this anomaly and it remains to be elucidated.

Whereas the meiotic LT/Sv phenotype comprises permanent $\mathrm{MI}$ arrest of the majority of oocytes, we did not observe such stable arrest during early embryonic mitoses. The explanation for this comes from the fact that in $\mathrm{Ml}$ arrested LT/Sv oocytes, at the time when SAC undergoes delayed inactivation, cytostatic factor (CSF) activity is already developed sustaining this arrest (Hirao \& Eppig 1997, Ciemerych \& Kubiak 1998). CSF is absent in early embryos, thus the delayed inactivation of 
SAC results only in prolonged prometaphase, but not in permanent arrest.

The knowledge gained on the meiotic LT/Sv phenotype offers also an explanation to the variability in the length of mitoses in LT/Sv embryos. A fraction of LT/SV oocytes ( $\sim 30 \%$ in our recent report (Hupalowska et al. 2008)) progresses through the first meiotic division unperturbed. Similarly, the length of mitoses of some LT/Sv embryos does not differ from the length of respective mitoses of wild-type embryos. In the current study, 13 out of 53 of one-cell and 6 out of 37 of the two-cell LT/Sv embryos completed mitosis until 115 and 78 min respectively, i.e. at the time when the average wild-type embryos completed their mitoses (this study). The remaining LT/Sv embryos completed divisions gradually, at varying times after NEBD. This phenomenon reflects the progressive exit from the first meiotic division of LT/Sv oocytes (Ciemerych \& Kubiak 1998), and a gradual release from meiotic MI arrest characteristic for oocytes with the $\mathrm{Mos}^{-1-}$ mutation introduced to an LT/Sv background (Hirao \& Eppig 1997).

In cells studied so far, SAC activity is transitory and is overcome if the perturbation persists over a critical period (Rieder \& Palazzo 1992, Rieder et al. 1994). By analogy, such SAC recovery might be crucial for the completion of the mitotic divisions of LT/Sv embryos. The support for such hypothesis comes from the presence of anomalies in chromosome separation occurring during mitoses of LT/Sv embryos, suggesting that the delayed anaphase might occur without SAC satisfaction. Alternatively, SAC activity might finally cease as SACactivating perturbation disappears. However, in such cases cleavage divisions should be unperturbed, yet we cannot exclude that more than one type of defect linked to SAC functioning acts in LT/Sv mice.

The high variability in the duration of embryonic mitoses could suggest that the relative success of reproduction of LT/Sv mice may rely upon those oocytes which pass only moderately perturbed $\mathrm{M}$-phases and thus segregate chromosomes properly. However, the prolongation of embryonic mitoses occurring not only in embryos originating from $\mathrm{Ml}$ but also from undisturbed MII oocytes clearly shows that normal completion of the first meiosis does not eliminate anomalies during the first mitosis. Therefore, the LT/Sv phenotype both in oocytes and embryos might comprise only meiotic elements of the cell-cycle control. Previous findings from our laboratories suggest that some meiotic pathways are functional also during the earliest cell-cycles of dividing embryos (Kubiak et al. 2008c). Thus, in LT/Sv mice, the defective meiotic elements of cell-cycle regulation could affect the SAC pathway not only during meiosis but also during the earliest embryonic divisions. Such defects, however, would disappear as soon as those meiotic components were replaced by mitotic ones, presumably as a result of embryonic genome expression. In LT/Sv embryos that successfully passed the critical meiotic and mixed meiotic/mitotic periods, further development could proceed unaffected. Alternatively, the defects may rely on maternal genes related to SAC function.

\section{Materials and Methods}

All animal studies presented were approved by Local Ethics Committee No. 1 in Warsaw, Poland and Regierungspraesidium in Freiburg, Germany, according to European Union Council Directive 86/609/EEC of 24 November 1986. All animals were raised on the premises.

\section{Collection, parthenogenetic activation of ovulated oocytes and in vitro culture of one- and two-cell embryos}

$\mathrm{F} 1(\mathrm{CBA} / \mathrm{H} \times \mathrm{C} 57 \mathrm{Bl} / 10)$ and $\mathrm{LT} / \mathrm{Sv}$ females were superovulated by injection of $10 \mathrm{IU}$ of eCG ('Folligon'; Intervet, Boxmeer, The Netherlands) and $10 \mathrm{IU}$ of hCG ('Chorulon'; Intervet) 48-52 h apart. Ovulated oocytes were collected 17-18 $\mathrm{h}$ after hCG injection and cumulus cells were removed with hyaluronidase (Sigma-Aldrich, 300 units/ml PBS, Biomed, Lublin, Poland). LT/Sv oocytes were classified as being at MI or MIl stage based on the absence or presence of the first polar body. For the parthenogenetic activation the oocytes were exposed to $8 \%$ ethanol (POCh, Gliwice, Poland) in $\mathrm{M} 2$ medium for $8 \mathrm{~min}$ at room temperature (Cuthbertson 1983), washed carefully and cultured in M2. Only those oocytes that extruded polar bodies and formed pronuclei were used for further analyses. Starting at $14 \mathrm{~h}$ after activation embryos were scored every 10 min to determine the timing of the NEBD and then collected for further analyses at different time after NEBD. Fertilized two-cell embryos were obtained from superovulated and mated females $49 \mathrm{~h}$ after hCG. Embryos were collected and cultured in M2 medium. Starting from $49 \mathrm{~h}$ after hCG embryos were observed every $10 \mathrm{~min}$ to determine the timing of NEBD and collected for further analyses at different times after NEBD.

\section{Collection and in vitro culture of fertilized one-cell embryos}

$\mathrm{BALB} / \mathrm{cPzhW}, \mathrm{C} 3 \mathrm{H} / \mathrm{W}, \mathrm{C} 57 \mathrm{BL} / \mathrm{Kw}, \mathrm{DBA} / 2 \mathrm{~W}$ (referred to throughout the text as $\mathrm{BALB} / \mathrm{C}, \mathrm{C} 3 \mathrm{H}, \mathrm{C} 57$ and DBA respectively), RI43, F1 (CBA/H×C57BI/10), and LT/Sv females were superovulated as described above and mated with F1 males. Fertilized one-cell embryos were obtained from oviducts $24 \mathrm{~h}$ after hCG injection and then cultured in M2 medium. Embryos subjected to analysis of the length of first mitotic division were scored every $10 \mathrm{~min}$ after NEBD. Embryos subjected to longterm culture were scored every $24 \mathrm{~h}$ until $120 \mathrm{~h}$ after hCG.

\section{Time-lapse recording of one- and two-cell embryos}

One-cell and two-cell embryos obtained after mating of F1 and LT/Sv females with F1 males were recorded during the first and the second mitotic division. For the time-lapse recording, the culture dish (glass-bottom dish; WillCo Wells BV, Amsterdam, 
The Netherlands) was placed in plastic chamber incubator XL (Zeiss; Carl Zeiss, Jena, Germany) mounted on a Zeiss Axiovert M200 inverted microscope (Zeiss, Carl Zeiss). The temperature in the incubator was maintained at $37^{\circ} \mathrm{C}$ by a Tempcontrol 37-2 digital (Zeiss, Carl Zeiss). The microscope was connected to AxioCam Mrm camera (Zeiss, Carl Zeiss). Image acquisition was performed using differential interface contrast (DIC) microscopy (30-400 ms illumination) every 5 min under the control of AxioVision 3.4 software (Zeiss, Carl Zeiss). Figures were assembled using Adobe Photoshop 7.0.

\section{Immunofluorescence}

The embryos were fixed and processed as previously described (Bouniol et al. 1995). $\alpha$-Tubulin was immunodetected with mouse MAB against $\boldsymbol{\alpha}$-tubulin (Sigma-Aldrich) followed by TRITC-labeled anti-mouse antibody (Jackson ImmunoResearch, West Grove, PA, USA). MAD2L1 was immunodetected by rabbit polyclonal antibody (gift from Dr K Wassmann; Wassmann et al. 2003), followed by FITC labeled anti-rabbit antibody (Jackson ImmunoResearch). Chromatin was visualized either with chromomycin A3 (Sigma-Aldrich) or propidium iodide (Vector Laboratories, Burlingame, CA, USA). The samples were mounted in Citifluor (Citifluor Ltd, London, UK) on glass slides and examined with laser scanning confocal microscope (Carl Zeiss). Figures were assembled using Adobe Photoshop 7.0.

\section{Statistical analysis}

Data were analyzed by $\chi^{2}$ test of independence, Fisher's exact probability test or $t$-test. Differences at $P<0.05$ were considered significant.

\section{Declaration of interest}

There is no conflict of interest that could be perceived as prejudicing the impartiality of the research reported.

\section{Funding}

This work was supported by grants from ARC (4900) and Ligue Contre le Cancer (Comité d'Ille-et-Vilaine et de la Vandée) to Jacek Z Kubiak.

\section{Acknowledgements}

The authors would like to thank Dr Katja Wassmann for anti MAD2L1 antibody and Anna Hupalowska for her help during the preparation of the figures.

\section{References}

Acquaviva C, Herzog F, Kraft C \& Pines J 2004 The anaphase promoting complex/cyclosome is recruited to centromeres by the spindle assembly checkpoint. Nature Cell Biology 6 892-898.

Albertini DF \& Eppig JJ 1995 Unusual cytoskeletal and chromatin configurations in mouse oocytes that are atypical in meiotic progression. Developmental Genetics 16 13-19.
Archacka K, Ajduk A, Pomorski P, Szczepanska K, Maleszewski M \& Ciemerych MA 2008 Defective calcium release during in vitro fertilization of maturing oocytes of LT/Sv mice. International Journal of Developmental Biology 52 903-912.

Artzt K, Calo C, Pinheiro EN, DiMeo-Talento A \& Tyson FL 1987 Ovarian teratocarcinomas in LT/Sv mice carrying t-mutations. Developmental Genetics 8 1-9.

Baker DJ, Jeganathan KB, Cameron JD, Thompson M, Juneja S, Kopecka A, Kumar R, Jenkins RB, de Groen PC, Roche P et al. 2004 BubR1 insufficiency causes early onset of aging-associated phenotypes and infertility in mice. Nature Genetics 36 744-749.

Baker DJ, Chen J \& van Deursen JM 2005 The mitotic checkpoint in cancer and aging: what have mice taught us? Current Opinion in Cell Biology 17 583-589.

Borsuk E \& Tarkowski AK 1989 Transformation of sperm nuclei into male pronuclei in nucleate and anucleate fragments of parthenogenetic mouse eggs. Gamete Research 24 471-481.

Bouniol C, Nguyen E \& Debey P 1995 Endogenous transcription occurs at the 1-cell stage in the mouse embryo. Experimental Cell Research 218 57-62.

Cheslock PS, Kemp BJ, Boumil RM \& Dawson DS 2005 The roles of MAD1, MAD2 and MAD3 in meiotic progression and the segregation of nonexchange chromosomes. Nature Genetics 37 756-760.

Chesnel F, Bazile F, Pascal A \& Kubiak JZ 2006 Cyclin B dissociation from CDK1 precedes its degradation upon MPF inactivation in mitotic extracts of Xenopus laevis embryos. Cell Cycle 5 1687-1698.

Chesnel F, Bazile F, Pascal A \& Kubiak JZ 2007 Cyclin B2/cyclin-dependent kinase1 dissociation precedes CDK1 Thr-161 dephosphorylation upon M-phase promoting factor inactivation in Xenopus laevis cell-free extract. International Journal of Developmental Biology 51 297-305.

Ciemerych MA 1995 Chromatin condensation activity and cortical activity during the first three cell cycles of a mouse embryo. Molecular Reproduction and Development 41 416-424.

Ciemerych MA \& Kubiak JZ 1998 Cytostatic activity develops during meiosis I in oocytes of LT/Sv mice. Developmental Biology 200 198-211.

Ciemerych MA, Tarkowski AK \& Kubiak JZ 1998 Autonomous activation of histone $\mathrm{H} 1$ kinase, cortical activity and microtubule organization in oneand two-cell mouse embryos. Biology of the Cell 90 557-564.

Ciemerych MA, Maro B \& Kubiak JZ 1999 Control of duration of the first two mitoses in a mouse embryo. Zygote 7 293-300.

Cuthbertson KS 1983 Parthenogenetic activation of mouse oocytes in vitro with ethanol and benzyl alcohol. Journal of Experimental Zoology 226 311-314.

Dobles M, Liberal V, Scott ML, Benezra R \& Sorger PK 2000 Chromosome missegregation and apoptosis in mice lacking the mitotic checkpoint protein Mad2. Cell 101 635-645.

Draetta G, Luca F, Westendorf J, Brizuela L, Ruderman J \& Beach D 1989 $\mathrm{Cdc} 2$ protein kinase is complexed with both cyclin A and B: evidence for proteolytic inactivation of MPF. Cell $\mathbf{5 6}$ 829-838.

Eppig JJ 1978a Developmental potential of LT/Sv parthenotes derived from oocytes matured in vivo and in vitro. Developmental Biology 65 244-249.

Eppig JJ $1978 b$ Granulosa cell deficient follicles: occurrence, structure, and relationship to ovarian teratocarcinogenesis in strain LT/Sv mice. Differentiation 12 111-120.

Eppig JJ, Wigglesworth K, Varnum DS \& Nadeau JH 1996 Genetic regulation of traits essential for spontaneous ovarian teratocarcinogenesis in strain LT/Sv mice: aberrant meiotic cell cycle, oocyte activation, and parthenogenetic development. Cancer Research 56 5047-5054.

Fang G, Yu H \& Kirschner MW 1998 The checkpoint protein MAD2 and the mitotic regulator CDC20 form a ternary complex with the anaphase-promoting complex to control anaphase initiation. Genes and Development 12 1871-1883.

Golas A, Dzieza A, Kuzniarz K \& Styrna J 2008 Gene mapping of sperm quality parameters in recombinant inbred strains of mice. International Journal of Developmental Biology 52 287-293.

Greda P, Karasiewicz J \& Modlinski JA 2006 Mouse zygotes as recipients in embryo cloning. Reproduction 132 741-748.

Guimaraes GJ, Dong Y, McEwen BF \& DeLuca JG 2008 Kinetochoremicrotubule attachment relies on the disordered $\mathrm{N}$-terminal tail domain of Hec1. Current Biology 18 1778-1784. 
Hampl A \& Eppig JJ 1995 Analysis of the mechanism(s) of metaphase I arrest in maturing mouse oocytes. Development 121 925-933.

Henery CC \& Kaufman MH 1992 Cleavage rate of diandric triploid mouse embryos during the preimplantation period. Molecular Reproduction and Development 32 251-258.

Henery CC \& Kaufman MH 1993a Cellular and nuclear volume of primitive red blood cells in digynic and diandric triploid and control diploid mouse embryos. European Journal of Morphology 31 237-249.

Henery CC \& Kaufman MH 1993b The cleavage rate of digynic triploid mouse embryos during the preimplantation period. Molecular Reproduction and Development 34 272-279.

Hirao Y \& Eppig JJ 1997 Analysis of the mechanism(s) of metaphase I arrest in strain LT mouse oocytes: participation of MOS. Development 124 5107-5113.

Hoffmann S, Tsurumi C, Kubiak JZ \& Polanski Z 2006 Germinal vesicle material drives meiotic cell cycle of mouse oocyte through the $3^{\prime}$ UTRdependent control of cyclin B1 synthesis. Developmental Biology 292 46-54.

Holloway SL, Glotzer M, King RW \& Murray AW 1993 Anaphase is initiated by proteolysis rather than by the inactivation of maturationpromoting factor. Cell 73 1393-1402.

Homer HA, McDougall A, Levasseur M, Yallop K, Murdoch AP \& Herbert M 2005 Mad2 prevents aneuploidy and premature proteolysis of cyclin B and securin during meiosis I in mouse oocytes. Genes and Development 19 202-207.

Hupalowska A, Kalaszczynska I, Hoffmann S, Tsurumi C, Kubiak JZ, Polanski Z \& Ciemerych MA 2008 Metaphase I arrest in LT/SV mouse oocytes involves the spindle assembly checkpoint. Biology of Reproduction 79 1102-1110.

Jeganathan KB \& van Deursen JM 2006 Differential mitotic checkpoint protein requirements in somatic and germ cells. Biochemical Society Transactions 34 583-586.

Kalab P, Kubiak JZ, Verlhac MH, Colledge WH \& Maro B 1996 Activation of p90rsk during meiotic maturation and first mitosis in mouse oocytes and eggs: MAP kinase-independent and -dependent activation. Development 122 1957-1964.

Kalitsis P, Earle E, Fowler KJ \& Choo KH 2000 Bub3 gene disruption in mice reveals essential mitotic spindle checkpoint function during early embryogenesis. Genes and Development 14 2277-2282.

Kallio M, Eriksson JE \& Gorbsky GJ 2000 Differences in spindle association of the mitotic checkpoint protein Mad2 in mammalian spermatogenesis and oogenesis. Developmental Biology 225 112-123.

Kaufman MH \& Howlett SK 1986 The ovulation and activation of primary and secondary oocytes in LT/Sv strain mice. Gamete Research 14 255-264.

Kaufman MH \& Speirs S 1987 The postimplantation development of spontaneous digynic triploid embryos in LT/Sv strain mice. Development 101 383-391.

Kubiak JZ \& Ciemerych MA 2001 Cell cycle regulation in early mouse embryos. In The Cell Cycle and Development. Novartis Foundation Sysmposium 237, pp 79-89. (discussion 89-99). Eds G Bock, G Cardew \& JA Goode. Chichester: John Wiley \& Sons Ltd.

Kubiak JZ, Bazile F, Pascal A, Richard-Parpaillon L, Polanski Z, Ciemerych MA \& Chesnel F 2008a Temporal regulation of embryonic M-phases. Folia Histochemica et Cytobiologica 46 5-9.

Kubiak JZ, Chesnel F, Richard-Parpaillon L, Bazile F, Pascal A, Polanski Z, Sikora-Polaczek M, Maciejewska Z \& Ciemerych MA $2008 b$ Temporal regulation of the first mitosis in Xenopus and mouse embryos. Molecular and Cellular Endocrinology 282 63-69.

Kubiak JZ, Ciemerych MA, Hupalowska A, Sikora-Polaczek M \& Polanski Z 2008 c On the transition from the meiotic to mitotic cell cycle during early mouse development. International Journal of Developmental Biology 52 201-217.

Lacefield S \& Murray AW 2007 The spindle checkpoint rescues the meiotic segregation of chromosomes whose crossovers are far from the centromere. Nature Genetics 39 1273-1277.

Lee GH, Bugni JM, Obata M, Nishimori H, Ogawa K \& Drinkwater NR 1997 Genetic dissection of susceptibility to murine ovarian teratomas that originate from parthenogenetic oocytes. Cancer Research $\mathbf{5 7}$ 590-593.
LeMaire-Adkins R \& Hunt PA 2000 Nonrandom segregation of the mouse univalent $X$ chromosome: evidence of spindle-mediated meiotic drive. Genetics 156 775-783.

LeMaire-Adkins R, Radke K \& Hunt PA 1997 Lack of checkpoint control at the metaphase/anaphase transition: a mechanism of meiotic nondisjunction in mammalian females. Journal of Cell Biology 139 1611-1619.

Li Y \& Benezra R 1996 Identification of a human mitotic checkpoint gene: hsMAD2. Science 274 246-248.

Ma J, Svoboda P, Schultz RM \& Stein P 2001 Regulation of zygotic gene activation in the preimplantation mouse embryo: global activation and repression of gene expression. Biology of Reproduction 64 1713-1721.

Maleszewski M \& Yanagimachi H 1995 Spontaneous and sperm-induced activation of oocytes in LT/Sv strain mice. Development, Growth and Differentiation 37 679-685.

Marangos P \& Carroll J 2008 Securin regulates entry into M-phase by modulating the stability of cyclin B. Nature Cell Biology 10 445-451.

Martin-Lluesma S, Stucke VM \& Nigg EA 2002 Role of Hec1 in spindle checkpoint signaling and kinetochore recruitment of Mad1/Mad2. Science 297 2267-2270.

Michel LS, Liberal V, Chatterjee A, Kirchwegger R, Pasche B, Gerald W, Dobles M, Sorger PK, Murty VV \& Benezra R 2001 MAD2 haploinsufficiency causes premature anaphase and chromosome instability in mammalian cells. Nature 409 355-359.

Mohammed AA, Karasiewicz J \& Modlinski JA 2008 Developmental potential of selectively enucleated immature mouse oocytes upon nuclear transfer. Molecular Reproduction and Development 75 1269-1280.

Musacchio A \& Salmon ED 2007 The spindle-assembly checkpoint in space and time. Nature Reviews. Molecular Cell Biology 8 379-393.

Niault T, Hached K, Sotillo R, Sorger PK, Maro B, Benezra R \& Wassmann K 2007 Changing mad2 levels affects chromosome segregation and spindle assembly checkpoint control in female mouse meiosis I. PLOS ONE 2 e1165.

Niemierko A 1975 Induction of triploidy in the mouse by cytochalasin B. Journal of Embryology and Experimental Morphology 34 279-289.

Nilsson J, Yekezare M, Minshull J \& Pines J 2008 The APC/C maintains the spindle assembly checkpoint by targeting Cdc20 for destruction. Nature Cell Biology 10 1411-1420.

Oh B, Hwang S, McLaughlin J, Solter D \& Knowles BB 2000 Timely translation during the mouse oocyte-to-embryo transition. Development 127 3795-3803.

O'Neill GT \& Kaufman MH 1987 Ovulation and fertilization of primary and secondary oocytes in LT/Sv strain mice. Gamete Research 18 27-36.

Pesin JA \& Orr-Weaver TL 2008 Regulation of APC/C activators in mitosis and meiosis. Annual Review of Cell and Developmental Biology 24 475-499.

Polanski Z 1997 Strain difference in the timing of meiosis resumption in mouse oocytes: involvement of a cytoplasmic factor(s) acting presumably upstream of the dephosphorylation of p34cdc2 kinase. Zygote $\mathbf{5}$ 105-109.

Polanski Z, Hoffmann S \& Tsurumi C 2005 Oocyte nucleus controls progression through meiotic maturation. Developmental Biology 281 184-195.

Ram PT \& Schultz RM 1993 Reporter gene expression in G2 of the 1-cell mouse embryo. Developmental Biology 156 552-556.

Rieder CL \& Palazzo RE 1992 Colcemid and the mitotic cycle. Journal of Cell Science 102 387-392.

Rieder CL, Schultz A, Cole R \& Sluder G 1994 Anaphase onset in vertebrate somatic cells is controlled by a checkpoint that monitors sister kinetochore attachment to the spindle. Journal of Cell Biology 127 1301-1310.

Sikora-Polaczek M, Hupalowska A, Polanski Z, Kubiak JZ \& Ciemerych MA 2006 The first mitosis of the mouse embryo is prolonged by transitional metaphase arrest. Biology of Reproduction 74 734-743.

Speirs S \& Kaufman MH 1988 Effect of exogenous hormones on the ovulation of primary and secondary oocytes in LT/Sv strain mice. Gamete Research 21 179-184.

Stevens LC \& Varnum DS 1974 The development of teratomas from parthenogenetically activated ovarian mouse eggs. Developmental Biology 37 369-380. 
Taylor SS \& McKeon F 1997 Kinetochore localization of murine Bub1 is required for normal mitotic timing and checkpoint response to spindle damage. Cell 89 727-735.

Tepperberg JH, Moses MJ \& Nath J 1999 Colchicine effects on meiosis in the male mouse. II. Inhibition of synapsis and induction of nondisjunction. Mutation Research 429 93-105.

Tsurumi C, Hoffmann S, Geley S, Graeser R \& Polanski Z 2004 The spindle assembly checkpoint is not essential for CSF arrest of mouse oocytes. Journal of Cell Biology 167 1037-1050.

Uhlmann F 2003 Chromosome cohesion and separation: from men and molecules. Current Biology 13 R104-R114.

Waksmundzka M, Krysiak E, Karasiewicz J, Czolowska R \& Tarkowski AK 1984 Autonomous cortical activity in mouse eggs controlled by a cytoplasmic clock. Journal of Embryology and Experimental Morphology 79 77-96.

Wang QT, Piotrowska K, Ciemerych MA, Milenkovic L, Scott MP, Davis RW \& Zernicka-Goetz M 2004 A genome-wide study of gene activity reveals developmental signaling pathways in the preimplantation mouse embryo. Developmental Cell 6 133-144.
Wassmann K, Liberal V \& Benezra R 2003 Mad2 phosphorylation regulates its association with Mad1 and the APC/C. EMBO Journal 22 797-806.

Winston NJ \& Maro B 1995 Calmodulin-dependent protein kinase II is activated transiently in ethanol-stimulated mouse oocytes. Developmental Biology 170 350-352.

Yamamoto A, Kitamura K, Hihara D, Hirose Y, Katsuyama S \& Hiraoka Y 2008 Spindle checkpoint activation at meiosis I advances anaphase II onset via meiosis-specific APC/C regulation. Journal of Cell Biology 182 277-288.

Yu HG, Muszynski MG \& Kelly Dawe R 1999 The maize homologue of the cell cycle checkpoint protein MAD2 reveals kinetochore substructure and contrasting mitotic and meiotic localization patterns. Journal of Cell Biology 145 425-435.

Received 16 January 2009

First decision 9 February 2009

Accepted 11 March 2009 